\title{
Lumen
}

Selected Proceedings from the Canadian Society for Eighteenth-Century Studies

\section{L'apparition du personnage du père dans le théâtre de Voltaire}

\section{Georges Bérubé}

Volume 17, 1998

Theatre of the world

Théâtre du monde

URI : https://id.erudit.org/iderudit/1012383ar

DOI : https://doi.org/10.7202/1012383ar

Aller au sommaire du numéro

Éditeur(s)

Canadian Society for Eighteenth-Century Studies / Société canadienne d'étude du dix-huitième siècle

ISSN

1209-3696 (imprimé)

1927-8284 (numérique)

Découvrir la revue

Citer cet article

Bérubé, G. (1998). L’apparition du personnage du père dans le théâtre de Voltaire. Lumen, 17, 91-101. https://doi.org/10.7202/1012383ar

Copyright (c) Canadian Society for Eighteenth-Century Studies / Sociéte canadienne d'étude du dix-huitième siècle, 1998
Ce document est protégé par la loi sur le droit d'auteur. L'utilisation des services d'Érudit (y compris la reproduction) est assujettie à sa politique d'utilisation que vous pouvez consulter en ligne.

https://apropos.erudit.org/fr/usagers/politique-dutilisation/ 


\section{L'apparition du personnage du père dans le théâtre de Voltaire}

Voltaire consacre les trois premiers articles de son Dictionnaire philosophique à une notion qui lui a toujours tenu à coeur, celle de la paternité. En effet, nous trouvons dans l'édition de Etiemble, laquelle nous présente une refonte des différentes éditions publiées par l'auteur de son vivant, ${ }^{1}$ les sujets suivants: 'Abbé,' 'Abraham' et 'Adam'. En guise d'introduction à la réflexion proposée ici, citons quelques lignes du premier article, 'Abbé,' dans lequel le philosophe s'adresse directement à son personnage, et indirectement à nous lecteurs, sur un ton humoristique:

Où allez-vous, monsieur 1'Abbé? etc. Savez-vous bien qu'abbé signifie père? Si vous le devenez, vous rendez service à l'État; vous faites la meilleure oeuvre sans doute que puisse faire un homme; il naîtra de vous un être pensant. Il y a dans cette action quelque chose de divin.

Mais si vous n'êtes monsieur l'abbé que pour avoir été tonsuré, pour porter un petit collet et un manteau court, et pour attendre un bénéfice simple, vous ne méritez pas le nom d'abbé. ${ }^{2}$

Etre père, même père en religion, comporterait toutes sortes d'obligations, comme nous le voyons bien ici! Si le sarcasme vise à abaisser les prétentions de ce genre de personnage, il ne diminue en rien l'importance du thème de la paternité, surtout dans ses rapports avec l'autorité et le droit, car ce thème s'impose partout dans l'oeuvre de Voltaire. Pensons à Zaïre, déchirée entre sa fidélité à son père Lusignan et celle à son amant Orosmane; pensons au jeune Candide qui a payé bien chèrement sa petite transgression - oh combien involontaire! - avec mademoiselle Cunégonde; pensons enfin aux déboires de l'Ingénu qui a eu tant de mal à trouver l'image d'un père juste dans la société française du dix-huitième siècle. ${ }^{3}$ Pour un homme comme Voltaire qui, toute sa vie durant, s'est beaucoup préoccupé de cette question, comment expliquer qu'il a différé si longtemps l'apparition du personnage du père dans son théâtre?

De fait, ce n'est que dans la tragédie de Brutus, commencée en Angleterre en 1728, que le père apparaît sur scène, soit une quinzaine 
d'années après le début de la rédaction d'Oedipe, et après quatre autres pièces, deux tragédies et deux comédies, qui ont été jouées dans les années $1720{ }^{4}$

Durant cette période, le dramaturge s'est contenté, semble-t-il, d'une simple évocation de l'image du père, que l'on songe aux récits de Jocaste et d'Oedipe qui retracent les grands bouleversements qui ont accompagné la disparition du roi Laïus (cf. IV,1), ou encore aux personnages utilisés comme figure substitutive du père, par exemple le Grand-Prêtre dans Oedipe qui se charge de prononcer la condamnation de celui qui a supprimé le roi légitime. Selon nous, cette absence du père dans le microcosme théâtral et le vide qu'elle entraîne ont une conséquence directe sur le plan dramaturgique, dans le sens qu'elle favorise le développement d'un autre personnage, celui du traître, qui trouve dans ce climat d'incertitude un champ d'activité on ne peut plus propice. Nous aurons l'occasion de revenir sur ce phénomène un peu plus tard.

Dès que l'on considère les comédies, l'Indiscret et la Fête de Bélébat, l'absence du père surprend encore davantage, car s'il y a un genre où celui-ci jouit depuis toujours d'un rôle privilégié, c'est bien dans la tradition de la comédie française héritée de Molière, qu'il s'appelle Gorgibus (les Précieuses Ridicules), Orgon (Tartuffe), ou Argan (le Malade Imaginaire). Voltaire, quant à lui, trouve le moyen de faire l'économie du père, dans l'Indiscret en le remplaçant par la mère et un vieux cousin influent du nom de Trasimon, dans la Fête de Bélébat, par le curé de Courdimanche, nom très évocateur de l'esprit de son créateur. Qu'està-dire sinon que Voltaire - ou peut-être était-il encore un peu trop Arouet comme le prétendait de façon dénigrante le chevalier de RohanChabot? - Voltaire, disions-nous, éprouvait une réticence certaine à créer un personnage qui, à cette époque, était pour lui fort problématique. La paternité d'Arouet père, la bâtardise prétendue de son fils, l'influence que tout cela a pu avoir sur les oeuvres de la période 17151730 , toutes ces questions pour le moins complexes ne sauraient être traitées dans le cadre de la courte étude que nous proposons maintenant. Tout au plus aimerions-nous poser une question à double volet: dans un premier temps, pourquoi, tout a coup en 1728, Voltaire sent-il le besoin de créer un tout nouveau personnage, comme cela avait été le cas pour Philoctète dans la tragédie d'Oedipe ? $^{5} \mathrm{D}^{\prime}$ ailleurs, Brutus - le personnage paternel qui a donné son nom à la tragédie - n'est que le premier d'une série, dont certains ont eu un retentissement considérable au Siècle des lumières: Lusignan dans Zaïre, Alvarez dans Alzire, Euphémon père dans l'Enfant Prodigue, Zopire dans Mahomet, pour n'en nommer que quelques-uns. Puis, dans un second temps, comment le dramaturge a-t-il mis en scène le personnage, à savoir quelles techniques dramaturgiques 
a-t-il développées pour ce faire, et finalement de quel contenu l'a-t-il investi?

Pourquoi 1728? Trois raisons principales semblent expliquer le fait que Voltaire ait soudain ressenti le besoin d'ajouter le père à sa liste de personnages théâtraux: primo, le 'second embastillement,' pour reprendre l'expression de René Pomeau, a l'effet d'un traumatisme dans la mesure où il rappelle à Voltaire la réalité qui fut sienne en 1717, celle qui lui avait appris une vérité simple et brutale: l'autorité et le droit ne protègent qu'une seule classe sociale, mais lui n'en fait pas partie. Secundo, la tactique qu'il avait mise au point à sa sortie de la Bastille en 1718 , soit celle qui consistait à se donner une nouvelle identité pour ensuite pactiser avec l'ennemi en tâchant de le flatter et de l'amadouer, se révèle inefficace et débouche en 1728 sur un échec. Tertio, il devient urgent désormais de se munir d'une nouvelle stratégie, si du moins Voltaire l'exilé souhaite réintégrer sa société et récupérer tout ce qu'il vient de perdre, stratégie que nous pourrions résumer ainsi: créer le personnage d'un père idéalisé qui garantit à tous ses sujets liberté et protection. Voyons les trois raisons dans l'ordre.

La première serait celle de la désillusion. A la suite de l'altercation avec Rohan-Chabot, Voltaire se retrouve une nouvelle fois à la Bastille, lui qui se voulait depuis dix ans déjà la coqueluche des salons les plus brillants de Paris! Tous ses efforts, ses rêves, pour ne pas dire ses ambitions, ont donc été vains. Nul doute que l'humiliation fut cinglante et qu'elle a profondément atteint celui qui croyait jusqu'alors faire partie de la bonne société, simplement parce qu'il la fréquentait et que partout la noblesse l'invitait. Mais il y a pis: l'affront se joint à l'injure lorsque le 'poète' - terme dénigrant employé par l'évêque de Blois et le maréchal de Villars ${ }^{6}$ - échoue dans sa tentative d'obtenir justice contre un noble, fût-il aussi peu respecté que Rohan-Chabot. Celui-ci ne l'avait-il pas fait rouer de coups? Et voici que la prétendue justice s'en prenait à Voltaire, la victime, et le faisait incarcérer sans procès. Tandis qu'au coupable, on ne fait absolument rien. De tous les grands que Voltaire avait côtoyés, amusés et divertis, aucun n'a levé le petit doigt pour appuyer publiquement le bon droit. La vérité éclate au grand jour: la solidarité qui liait les gens de la noblesse entre eux primait sur toute notion de justice sociale. Privé ainsi de ses protecteurs et de la sécurité qu'il croyait avoir acquise depuis ses démêlés de 1718, Voltaire opte pour l'exil afin de réévaluer ses chances de réussite dans une société à ce point sclérosée. La morale de cette histoire pourrait se formuler de la sorte: on n'affronte jamais ouvertement une autorité qui ne favorise que les grands.

Voilà donc que la bastonnade et l'emprisonnement déclenchent une réflexion parfois assez amère sur la justice arbitraire qui règne en France à cette époque. N'est-ce pas celle décrite dans Le Monde comme il va, celle 
du jeune magistrat étourdi à qui l'État vend sa charge? On apprend à Babouc qu'en effet 'jamais ceux qui ont vieilli dans les emplois laborieux et subalternes ne parviennent aux dignités. Ce jeune homme a une grande charge, parce que son père est riche, et qu'ici le droit de rendre la justice s'achète comme une métairie. ${ }^{77}$ La justice représente donc une valeur monnayable que le roi trafique avec les nobles. Comment faire pour que la situation s'améliore? Ces problèmes, nous les retrouvons abordés dans les discussions entre Brutus et le représentant de la monarchie tyrannique. Plus qu'une simple discussion toutefois, car elle oblige le dramaturge à opérer une véritable révolution théâtrale en incarnant la justice tant souhaitée dans le personnage intègre du père.

La seconde raison découle de la première, car qui dit désillusion laisse supposer son contraire, qu'il y eut d'abord illusion. Ayant déjà souffert de l'arbitraire de l'autorité lorsque le Régent l'avait fait emprisonner sur lettre de cachet en 1717-1718, Voltaire en était ressorti avec ce que nous proposons d'appeler une tactique qui lui permettait de mieux se protéger tout en avançant dans le monde. Il se fait désormais appeler Monsieur de Voltaire, signifiant par là sa volonté de faire oublier le prisonnier Arouet, ainsi que son désir d'appartenir à cette classe qui l'avait persécuté. Autrement dit, il voulait se rallier à la cause du Régent pour ne plus être considéré comme un élément perturbateur et dangereux, mais un sympathisant qui méritait la considération des grands. Le triomphe d'Oedipe, la médaille d'or offerte par le Régent, la dédicace qu'accepte sa mère, la Princesse palatine, le succès de la Henriade, autant de signes d'une notoriété publique qui résulte d'une vie sociale très active en compagnie des gens les plus prestigieux du royaume. Une telle adulation a pu lui faire croire que son personnage jouissait dorénavant d'une immunité à toute épreuve. Rien n'illustre mieux cette certitude que la Fête de Bélébat, récit d'un divertissement dialogué que l'auteur expédia directement à la reine de France: encouragé et recherché par une aristocratie enjouée et licencieuse, Voltaire se met lui-même en scène. Il distribue à chaque noble présent les hommages les plus flatteurs, tout en s'octroyant la couronne - de lauriers s'entend - quand il détrône le curé de Courdimanche pour prendre sa place à la fin. Voltaire est acclamé roi des plaisirs, reconnu souverain par tous les gens à la mode! Dans ces conditions, est-il étonnant qu'il se soit laissé prendre à son propre jeu littéralement?

Comment cette tactique a-t-elle influencé son théâtre? L'organisation des forces théâtrales reflète-t-elle sa nouvelle vision du monde? Dans la mesure où Voltaire veut apaiser le pouvoir, il tente dans ses pièces de le ménager, de ne jamais le prendre à parti directement. Le détenteur de l'autorité est-il impliqué dans un crime ou abus de pouvoir quelconque, le dramaturge s'efforce d'en minimiser l'importance, voire de l'excuser, 
en ayant recours au personnage du traître sur qui retombe l'odieux. Par exemple, un confident malhonnête peut, par une manipulation éhontée, induire son maître en erreur ou le pousser carrément à commettre un crime. Dans le cas d'Artémire, le tyran Cassandre est en grande partie disculpé parce que constamment trompé par son ignoble ministre Pallante; dans Mariamne, le roi Hérode cède à la douleur et sombre dans la folie, parce qu'il a fait exécuter la reine, faussement accusée par l'intrigante Salome et son comparse Mazaël. L'avantage pour Voltaire paraît clair: le pouvoir demeure toujours indemne.

Par le moyen de cette tactique, le dramaturge pactise avec le pouvoir en tentant de se maintenir dans ses bonnes grâces. Bien entendu, les crimes de Cassandre et de Hérode n'en sont pas moins réels et les suites, non moins tragiques, mais Voltaire, en bon élève de la casuistique jésuite, exonère le coupable en contruisant un univers rempli d'accommodements taillés sur mesure. Les confesseurs des rois n'agissaient pas autrement et certains s'en sont trouvés fort bien.

Le système a fonctionné à merveille tant que Voltaire a eu l'impression de voir tous ses projets réussir. Damis, dans l'Indiscret, lui sert de porteparole quand il déclare sans ambages que, dans ce bas monde, l'hypocrisie est la règle, que le monde appartient aux joueurs les plus habiles; ${ }^{8}$ dans la Fête de Bélébat, Voltaire le poète usurpe le pouvoir grâce à la ruse de sa plume. Pourtant, l'illusion se dissipe dès qu'elle se bute à la dure réalité.

On imagine l'impact de l'incident Rohan sur une telle conception du monde! Puisque le pouvoir et la responsabilité sont dissociés, le souverain garde malgré tout son innocence, mais où faut-il chercher le responsable, et comment lui faire avouer sa culpabilité? Le chevalier de Rohan jouait de toute évidence ici le rôle du traître - même l'autorité reconnaissait implicitement qu'il n'est pas permis d'attaquer un autre homme en pleine rue, fût-il poète - mais il n'existait hélas aucun dramaturge avec un pouvoir politique suffisant pour l'obliger à rendre compte de ses actes. Voltaire comprenait que si le monde était un théâtre, ce n'était pas lui qui tirait les ficelles comme à Bélébat, qu'en fait le dénouement allait s'écrire indépendamment de sa volonté. Sa conception théâtrale, en tant que tactique d'avancement social, venait d'échouer lamentablement. Ne restait plus qu'à se désister, tourner la page et tenter sa chance ailleurs, en l'occurrence en Angleterre.

Ce qui nous amène à la troisième raison qui explique l'apparition du père, soit la nécessité d'élaborer un nouveau système, une tactique plus efficace, qui permettrait à Voltaire un retour en France, non comme un exilé défait et repentant, mais plutôt comme un philosophe aguerri et prêt à livrer bataille. Le séjour chez les Anglais favorise la recherche et la mise au point de ce nouveau système, dans lequel le personnage du 
père détient l'autorité et assume réellement la responsabilité de ses actes. Il sera à l'image du roi d'Angleterre décrit dans les Lettres philosophiques, c'est-à-dire 'un Prince, tout puissant pour faire du bien [mais qui] a les mains liées pour faire le mal. ${ }^{9}$ En somme, Voltaire songe à un père idéalisé qui garantit à tous ses sujets, quelle que soit leur classe sociale, liberté et protection, ainsi qu'une égalité absolue devant la loi. Louis Dubois, dans une notice sur la tragédie de Brutus, fait cette remarque :

Le séjour de Voltaire en Angleterre, la vue d'un peuple libre, et sa comparaison avec l'état où se trouvait la France livrée au bon plaisir des ministres, des courtisans et des courtisanes en crédit, tournèrent vers les objets philosophiques et les avantages du régime libéral les idées du poète qui avait reçu dans sa patrie qu'il honorait les disgrâces de l'exil et de deux emprisonnements à la Bastille. Brutus fut l'un des fruits les plus remarquables du voyage de Londres (nous soulignons). ${ }^{10}$

Les courtisans comme Rohan-Chabot n'auraient plus, dans cette nouvelle conception de l'État, le crédit ou la force de nuire, ni de corrompre le père, voire $d$ 'influencer son jugement, de sorte que la justice dépendrait désormais de la seule intégrité du père-souverain.

Après avoir discuté les raisons qui ont pu pousser Voltaire à créer le personnage du père, voyons comment il s'y est pris, à savoir les techniques dramaturgiques mises en oeuvre, ainsi que le contenu dont il a cru bon l'investir.

Sur le plan de la dramaturgie, disons tout d'abord qu'il y a une rupture avec les oeuvres antérieures. Dans Brutus, aucun personnage secondaire n'intervient pour annoncer l'arrivée du héros, ce qui constituait une technique fréquemment utilisée dans le théâtre classique. Au contraire, Brutus paraît au milieu des sénateurs romains et, dès le lever $\mathrm{du}$ rideau, il prend la parole en tant que consul de Rome. Dans ses premières tragédies, Voltaire retardait le plus longtemps possible l'entrée en scène du détenteur du pouvoir: dans Oedipe, celui-ci arrive à la fin de l'acte I; Cassandre, dans Artémire, est absent jusqu'à l'acte IV et Hérode dans Mariamne, jusqu'à l'acte III. L'arrivée tardive du souverain prend tout son sens à la lumière de ce qui a été avancé plus tôt: il s'agit d'une absence qui favorisait les activités subversives du traître et qui préservait l'intégrité toute relative du pouvoir - dans la mesure où celui-ci les ignorait. Dans Brutus, ce vide est comblé d'entrée de jeu par la présence fortement marquée du père, qui occupe une place à ce point importante qu'elle signifie de manière non équivoque qu'il est le seul à exercer le pouvoir. Ce changement apporté par le dramaturge à la structure même de la pièce montre l'importance qu'il accorde maintenant à ce nouveau père-souverain. 
En ce qui concerne la mise en scène que Voltaire voulait frappante dans sa solennité, il recommande que le spectateur voie dans le fond 'le temple du Capitole' et en premier plan 'une partie de la maison des consuls':

Les sénateurs sont assemblés entre le temple et la maison, devant l'autel de Mars. Brutus et Valérius Publicola, consuls, président à cette assemblée: les sénateurs sont rangés en demi-cercle. Des licteurs avec leurs faisceaux sont debout derrière les sénateurs. ${ }^{11}$

Et pour ajouter encore plus d'éclat à la scene, ceux-ci sont vêtus de robes rouges. Ces 'audaces ${ }^{12}$ peuvent paraître bien anodines de nos jours, mais à l'époque, sur une scène encombrée de spectateurs, elles représentaient un effort de la part du dramaturge de revêtir le pouvoir d'une auréole de gloire. De plus, la présence des sénateurs avec Brutus à leur tête devenait le signe visible que le pouvoir n'allait laisser au traître qu'une marge de manoeuvre bien restreinte.

Outre la structure renouvelée, Voltaire cherche à donner le plus de poids possible à l'apparition de la figure paternelle en lui faisant prononcer d'emblée un discours plein des 'nobles et fiers accents de la liberté,' l'expression est de Louis Dubois. De nouveau, il s'agit d'un changement radical, car dans les tragédies précédentes le dramaturge s'était borné à suivre les règles classiques qui régissaient l'exposition de l'action: deux personnages se renseignaient l'un l'autre sur l'état des forces en présence. L'exposition ainsi conçue consistait en une transmission d'informations, avec d'éventuels projets qu'un des perșonnages allait chercher à réaliser. Tandis que dans Brutus, la communication $d$ 'informations existe bel et bien, mais elle est prise en charge par une rhétorique enflamée, chargée d'idéologie, René Pomeau parle d'un 'républicanisme, s'exhalant en tirades à effet, en formules à l'emportepièce, ${ }^{\prime 13}$ qui tranche nettement avec tout ce que Voltaire avait osé écrire pour le théâtre jusqu'alors, les quelques vers d'Oedipe exceptés. D'ailleurs, l'intensité des harangues fut telle que, pendant la Révolution, lors d'une reprise de la pièce, elle eut un franc succès auprès des républicains avides d'émotions fortes. Qu'y a-t-il dans ce discours initial?

Écoutons l'éloquence des premiers vers qui donnent le ton à la pièce:

Destructeurs des tyrans, vous qui n'avez pour rois

Que les dieux de Numa, vos vertus et nos lois,

Enfin notre ennemi commence à nous connaître.

Ce superbe Toscan qui ne parlait qu'en maître,

Porsenna, de Tarquin, ce formidable appui, 
Ce tyran, protecteur d'un tyran comme lui ...

Respecte le sénat et craint un peuple libre. ${ }^{14}$

Il y a là une affirmation éclatante, placée comme elle l'est au tout début de la pièce, de la primauté des lois sur la volonté arbitraire du tyran que la république venait de renverser. Ne peut-on pas y lire en filigrane aussi la devise du nouveau Voltaire, le philosophe revenu d'exil, qui condamne le traitement injuste dont il a été victime? Brutus termine son discours ainsi en faisant allusion à l'ambassadeur du roi Tarquin : 'L'ennemi du sénat connaîtra qui nous sommes, / Et l'esclave d'un roi va voir enfin des hommes. ${ }^{15}$ La scène se clôt sur la reconnaissance de Brutus comme père de la liberté romaine, de sorte que le père idéalisé paraît dans toute sa gloire: $c^{\prime}$ est celui qui peut rappeler même aux tyrans que les hommes sont des êtres libres et égaux devant la loi.

Dans la scène suivante, une discussion mouvementée s'engage entre l'ambassadeur Arons et Brutus quant à savoir si un peuple peut rompre le lien qui l'attache à son roi. Aux interrogations répétées d'Arons qui se veulent comme autant de reproches:

Quels dieux ont donc changé les droits des souverains?

Quel pouvoir a rompu des noeuds jadis si saints?

Qui du front de Tarquin ravit le diadême?

Qui peut de vos serments vous dégager? ${ }^{16}$

Brutus répond que les crimes du tyran ont seuls rompu le pacte qui unissait le peuple à son souverain. Car, ajoute-t-il, les Romains ont fait serment d'obéissance et non d'esclavage. Comment ne pas entendre dans ce discours une résonance de Voltaire défiant le pouvoir arbitraire qui l'a humilié? Il destitue le souverain coupable bien qu'il soit encore en théorie légitime, et transfert son pouvoir à un père créé de toutes pièces:

Devant ces mêmes dieux, il [le roi] jura d'être juste.

De son peuple et de lui tel était le lien:

Il nous rend nos serments lorsqu'il trahit le sien;

Et dès qu'aux lois de Rome il ose être infidèle,

Rome n'est plus sujette, et lui seul est rebelle. ${ }^{17}$

Ces quelques passages suffisent sans doute pour montrer la force de caractère dont Voltaire a investi ce père idéal. Il affiche son intégrité comme un drapeau et rien ni personne ne peut lui porter ombrage.

Brutus est la première d'une série de figures paternelles créées par Voltaire au cours des années 1730, mais la plus connue demeure Lusig- 
nan dans Zaïre. Si la durée de sa présence sur scène est plus brève que celle de Brutus, elle est par ailleurs mémorable. Les spectateurs se rappelaient facilement quelques-unes de ses répliques qui ont grandement contribué à la réputation de la pièce. ${ }^{18} \mathrm{Ce}$ qui importe pour nous en revanche, c'est l'importance que le personnage avait pour son créateur. Toute sa vie, sur ses nombreux théâtres privés, Voltaire a repris ce rôle et, de l'avis de plusieurs témoins, il le jouait avec intelligence et beaucoup de sensibilité. Eva Jacobs, dans l'introduction à son édition critique, écrit:

It is generally thought that Lusignan was Voltaire's favourite acting part. At any rate, the number of known private performances of Zaïre he gave suggests that ... he shared the public's fondness for this particular play. [When Voltaire] played the part of Lusignan on his private stage ... he acted with a depth of feeling and pathos that frequently made his audience weep with him. ${ }^{19}$

Comment expliquer sa prédilection pour Lusignan, si ce n'est qu'il représentait ce père idéal, intègre et malheureux?

En guise de conclusion, disons que Voltaire se croyait sans doute victime, et du père Arouet qui avait cherché souvent à discipliner son fils un peu trop frondeur, et du Régent/Roi qui l'avait fait incarcérer par deux fois. Pour bien annoncer sa rupture avec ces deux instances paternelles, Voltaire rejette son père biologique, se dit bâtard et se crée une nouvelle identité avec un nom bien à lui, puis défie le 'père' politique en transférant, sur le plan de la représentation théâtrale, le pouvoir du tyran Tarquin - roi héréditaire et somme toute légitime - à Brutus, nouveau père de la liberté romaine. Cette notion de la paternité n'a plus grand chose à voir avec le physique, puisqu'elle se veut avant tout idéologique et symbolique. Un self-made-man comme Monsieur de Voltaire - et c'est ce que signifiait sa riposte au chevalier de Rohan - a bien le droit de se donner le père qui lui convient.

Notons finalement que Brutus, comme c'est le cas pour les autres pères qui le suivront, perd ses enfants qui tous deux sont éxécutés pour trahison. En revanche, il s'entoure d'enfants adoptifs qui reprennent le flambeau de son idéal de liberté, et c'est ce qui importe davantage pour l'avenir de Rome. 


\section{Endnotes}

1 Basée sur le texte établi par Raymond Naves, l'édition de Etiemble offre l'avantage d'une vision synthétique, alors que l'édition originale a subi de nombreux remaniements, sans parler des additions et des variantes. Pour un aperçu de l'évolution du Dictionnaire philosophique et des Questions sur l'Encyclopédie, cf. les remarques de R. Naves (Paris: Éditions Garnier Frères, 1967) xxiii-xxvi; pour une histoire de l'oeuvre, cf. l'édition du Dictionnaire publiée sous la direction de Christiane Mervaux, dans Oeuvres complètes de Voltaire (Banbury: Voltaire Foundation, 1994) 35: 20-60.

2 Etiemble, 1.

3 Les personnages des contes, même ceux qui se veulent les plus pacifiques, affrontent inévitablement diverses instances d'un pouvoir arbitraire. Micromégas a eu maille à partir avec 'le muphti de son pays, grand vétillard, et fort ignorant' qui fait appel à des jurisconsultes tout aussi bêtes que lui; Zadig, quant à lui, souffre de l'envie et de la jalousie, d'abord des courtisans de la cour, puis du souverain.

4 Il s'agit des tragédies, Artémire (1720), Hérode et Mariamne (1724), devenue par la suite Mariamne (1725); de la comédie, l'Indiscret (1725), et du divertissement, la Fête de Bélébat (1725).

5 Voir notre article, 'Le personnage de Philoctète dans l'Oedipe de Voltaire: un signe avant-coureur,' Lumen 13 (1994): 61-71.

6 Voici ces deux opinions fort révélatrices de l'aristocratie de cette époque: 'Ces coups, s'agissant d'un poète,' remarque René Pomeau, 'certains les trouvent naturels. "Nous serions bien malheureux si les poètes n'avaient point d'épaules," a dit l'évêque de Blois, un Caumartin ... Le maréchal de Villars, comme on lui disait que "le chevalier de Rohan ne devait pas donner des coups de bâton, que cela est contre les lois," répond: "Mais c'est un poète."'; D'Arouet à Voltaire, Voltaire en son temps (Banbury: Voltaire Foundation, 1985) 1: 206.

7 Le Monde comme il va, dans Voltaire, romans et contes, préface et notes de René Pomeau (Paris: Garnier-Flammarion, 1966) 99.

8 Damis s'exprime ainsi au début de la pièce:

je sais bien qu'en ce monde

Il faut, pour réussir, une adresse profonde.

Hors dix ou douze amis à qui je puis parler,

Avec toute la cour je vais dissimuler.

Oeuvres complètes de Voltaire, éd. L. Moland (Paris: Éditions Garnier Frères, 1877) 2: 250. Dans cette étude, toutes les citations tirées des textes théâtraux renvoient désormais à cette edition.

9 Voltaire, Lettres philosophiques, éd. Raymond Naves (Paris: Éditions Garnier Frères, 1964) 34.

10 Oeuvres complètes de Voltaire, éd. Arguis, Clogenson et al. (Paris: Librairie Universelle de P. Mongie, 1828) 3: 359.

11 M. 2:327.

12 Dans le 'Discours sur la tragédie' publié avec Brutus, Voltaire s'explique sur ses audaces: 'Plus on veut frapper les yeux par un appareil éclatant, plus on s'impose la nécessité de dire de grandes choses; autrement on ne serait qu'un décorateur, et 
non un poète tragique ... Pour moi, j'avoue que ce n'a pas été sans quelque crainte que j'ai introduit sur la scène française le sénat de Rome en robes rouges, allant aux opinions. Je me souvenais que lorsque j'introduisis autrefois dans Oedipe un choeur de Thébains qui disait: O mort, nous implorons ton funeste secours! / O mort, viens nous sauver, viens terminer nos jours! la parterre, au lieu d'être frappé du pathétique qui pouvait être en cet endroit, ne sentit d'abord que le prétendu ridicule d'avoir mis ces vers dans la bouche d'acteurs peu accoutumés, et il fit un éclat de rire. C'est ce qui m'a empêché, dans Brutus, de faire parler les sénateurs' (M. 2:320-21).

13 Pomeau, 266. Pour une discussion approfondie du républicanisme de Voltaire dans Brutus, voir Ronald Ridgway, 'La Propagande philosophique dans les tragédies de Voltaire,' Studies on Voltaire and the Eighteenth Century 15 (1961): 71-81; voir aussi Thomas M. Carr, Jr., 'Dramatic structure and philosophy in Brutus, Alzire and Mahomet,' Studies on Voltaire and the Eighteenth Century 143 (1975): 725.

14 M. 2:327.

15 Ibid., 328.

16 Ibid., 330.

17 Ibid., 330.

18 Outre la reconnaissance de Zaïre provoquée par la croix de sa mère, on se souvient de ces vers de Lusignan:

Dissipez mes soupçons, ôtez-moi cette horreur,

Ce trouble qui m'accable au comble du bonheur.

Toi qui seul as conduit sa fortune et la mienne,

Mon Dieu qui me la rends, me la rends-tu chrétienne? (M, 2:578)

19 Eva Jacobs, 'Introduction' à son édition critique de Zaïre, Oeuvres complètes de Voltaire (Oxford: Voltaire Foundation, 1988) 8: 291-292, 318. 